\title{
A novel combinatorial treatment option for metastatic uveal melanoma
}

\author{
Dudi Shneor ${ }^{1,2}$, Shay Tayeb ${ }^{1,3}$, Jacob Pe'er ${ }^{2}$, Hanna Voropaev ${ }^{1,2}$, Maria \\ Gimmelshein ${ }^{1,2}$, Nathalie Cassoux ${ }^{4,5}$, Alik Honigman ${ }^{1,3, *}$ and Shahar Frenkel ${ }^{2, *}$ \\ ${ }^{1}$ Department of Biochemistry and Molecular Biology, IMRIC, The Hebrew University-Hadassah Medical School, Jerusalem, \\ Israel \\ ${ }^{2}$ Department of Ophthalmology, Hadassah-Hebrew University Medical Center, Jerusalem, Israel \\ ${ }^{3}$ Hadassah Academic College, Jerusalem, Israel \\ ${ }^{4}$ Department of Ocular Oncology, Institut Curie, Paris, France \\ ${ }^{5}$ Université Paris V Descartes, Paris, France \\ "These authors have contributed equally to this work \\ Correspondence to: Shahar Frenkel, email: shahar.frenkel@gmail.com \\ Keywords: cancer; chemotherapy; replicative competent retroviruses (RCR); CREB; combinatorial targeted treatment \\ Received: February 16, $2018 \quad$ Accepted: April 28, $2018 \quad$ Published: May 25, 2018
}

Copyright: Shneor et al. This is an open-access article distributed under the terms of the Creative Commons Attribution License 3.0 (CC BY 3.0), which permits unrestricted use, distribution, and reproduction in any medium, provided the original author and source are credited.

\section{ABSTRACT}

Uveal melanoma (UM) is the most frequent intraocular tumor in adult patients. When metastases occur, systemic therapy with alkylating agents (fotemustine or dacarbazine (DTIC)) has shown only modest efficacy. The common chemotherapeutic drug doxorubicin (DOX) is not used to treat metastatic UM (mUM). To expand the chemotherapeutic arsenal for mUM, we tested the effect of DOX on UM cell mortality. We have previously shown that CREB knockdown enhances sensitivity to DOX. UM cells infected with recombinant MuLV-based replicative competent retroviruses (RCR) expressing shRNA targeting CREB were co-treated with either DTIC or DOX. We found that CREB knockdown increases the sensitivity of these cells to both DOX and DTIC in normoxia and more so in hypoxia as measured by cell survival and Caspase 3 activation. The ability to combine CREB knockdown by infection with the RCR recombinant virus which preferentially infects replicating tumor cells and chemotherapy to achieve the same amount of cell death in lower concentrations may result in fewer side effects of the drugs. This combination is a possible new treatment for mUM.

\section{INTRODUCTION}

Uveal melanoma (UM) is the most frequent intraocular tumor in adult patients. Up to $50 \%$ of patients will develop metastases [1], of which $80 \%$ die in the first year, and $92 \%$ within the first two years [2]. Systemic therapy with alkylating agents, i.e., fotemustine or dacarbazine (DTIC), have shown only modest efficacy [3, 4]. Consequently, because of the limited efficacy of current treatments, new therapeutic strategies need to be developed.

One of the primary means by which UM cells evade treatment-induced apoptosis is by up-regulation of members of the pro-survival Bcl-2 family proteins such as Bcl-2 and Bcl- $\mathrm{X}_{\mathrm{L}}[5,6]$. Indeed, up to $82 \%$ (range: $44 \%-$ $100 \%$ ) of human UMs are characterized by elevated Bcl-2 levels [7-11] without any prognostic impact [7, 10-12].

The transcription factor cyclic AMP-responseelement (CRE) binding protein (CREB) was previously found by our team to suppress Hepatocellular Carcinoma (HCC) cell death under hypoxic conditions. Overexpression of positive dominant CREB300/310 prevented cell death in hypoxia [13]. On the other hand, CREB knockdown increased HCC cell sensitivity to hypoxia as well as to doxorubicin (DOX) in normoxia and hypoxia [14]. Recently, it was demonstrated that CREB 
blockade by decoy oligonucleotides functionally inhibited transactivation of CREB, and significantly increased radio-sensitivity of multiple human cancer cell lines [15]. Overexpression of CREB decreases expression of the pro-death Bim protein and inhibits the sequestration of Bim protein from tubulin molecules, thereby protecting cells from apoptosis [16]. Additionally, activation of Bcl-2 expression by CREB promotes cell survival, while Trichosanthin's inhibition of CREB's activation of cell cycle regulatory proteins such as cyclin A resulted in cell cycle arrest [17]. CREB is also involved in the apoptotic effect of DOX, where DOX-induced p38 activation can suppress the PKA pathway, preventing activation of CREB and thus leading to downregulation of Bcl-2 and as a result to enhanced apoptosis [18]. Sayan et al found that combining inhibition of CREB phosphorylation with DOX treatment was significantly more effective in mesotheliomas [19]. These findings along with our ability to increase tumor cell sensitivity to DOX led us to test if this combination is also effective in UM.

Although DOX is a well-accepted chemotherapeutic agent in a variety of metastatic neoplastic diseases [20], it is hardly used for metastatic uveal melanoma. An extensive literature review identified only a single case report by Brasiuniene et al. that describes the use of DOX in combination with two other chemotherapies and interferon $\alpha$ for metastatic UM with liver lesions after other treatments have failed. This therapy led to a partial response and was later followed by resection of the metastases [21]. Recently, Latorre and colleagues used gold nanoparticles (GNPs) for targeted delivery of a high concentration of DOX to UM cell lines. They demonstrated that modified GNPs could be functionalized to increase the efficacy of cancer therapeutics and may further reduce toxicity by increasing targeted delivery towards malignant cells [22].

In this work, we infected the cells with a MuLVbased recombinant replication competent retrovirus (RCR) [23] which expresses shRNA targeting CREB (vACE-CREB) [14] prior to treatment of the tumors with chemotherapeutic drugs. The advantage of using this vector is that MuLV retroviruses infect only replicating cells such as tumor cells, generating a stably integrated provirus in the cells. These cells produce viral particles expressing shRNA targeting CREB that will spread within the tumor [14].

Solid tumors grow faster than they can attract blood vessels into them generating an ongoing formation of hypoxic regions within tumors. In these regions, tumor cells are too far from the present vessels, and thus oxygen and drugs do not reach them. This poses a problem in killing solid tumors: these areas are radio-resistant due to the lack of active oxygen radicals, and are chemoresistant due to the lack of tumoricidal concentrations of the drugs [24]. Unlike normal tissues, solid tumors are more resistant to hypoxia. CREB was found to play a pivotal role in the response of cells to hypoxia [13] along with the hypoxia-inducible factors 1 and 2 (HIF-1 and HIF-2). To overcome the problem of these resistant regions to chemotherapeutic treatments, we constructed additional RCR vectors expressing shRNA targeting the three hypoxia-response regulating genes. We have shown a decreased survival in hypoxia of the recombinant RCR infected HCC cells and an increase in the sensitivity of these infected cells in hypoxia to DOX [14].

Targeting the unique property of ongoing generation of hypoxic areas in tumors by the RCRs along with the preference for replicating cells, will preferentially affect tumors in a double-targeting mechanism. We demonstrate here that infecting UM cells with vACE-CREB sensitizes them to treatment with DOX and DTIC.

The increased sensitivity to chemotherapy in the RCR-infected cells in vitro may set the basis for a more efficient combined treatment for metastatic UM.

\section{RESULTS}

\section{Infectivity of the recombinant RCRs in UM cell lines}

To construct a MuLV replicating viral vector that expresses shRNA targeting CREB (Figure 1) the IRESGFP DNA fragment in vACE-GFP [23] was replaced by the $\mathrm{H} 1$ promoter driving the shRNA sequences targeting CREB (pACE-CREB) or expressing a non-target sequence (pACE-NT) as previously described [14].

The titer of the viral preparations was defined by comparison of qPCR of the env gene to RNase P (a

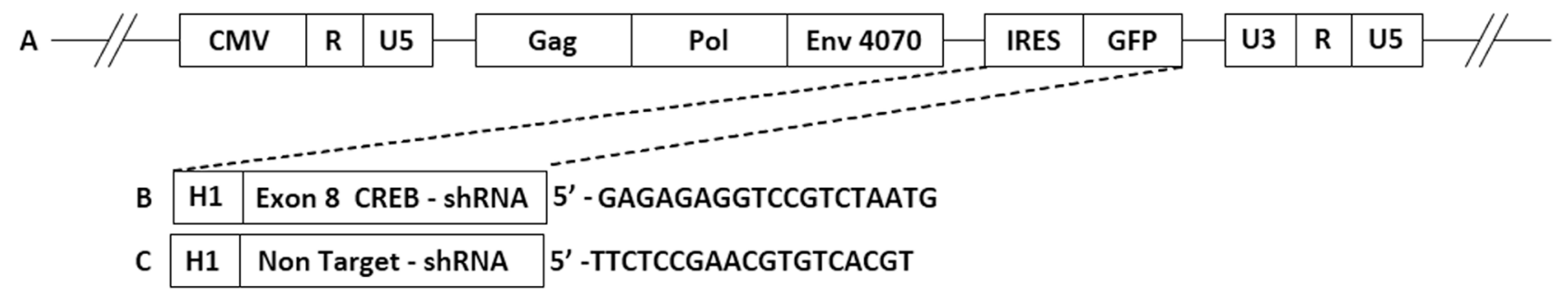

Figure 1: A schematic presentation of the various RCRs. (A) The provirus construct of pACE-GFP. (B, C) Replacement of the IRES-GFP sequences with an $\mathrm{H} 1$ promoter driving the transcription of shRNAs. Sequences coding for the shRNA are specified. 
single copy gene per cell) in cells 48 hours after infection. This method quantifies the infective particles in the viral preparations.

GFP fluorescence from cells infected with vACEGFP served to determine the kinetics of spread of the virus in Mel 270 and OMM2.5 cells in culture. The efficiency of infectivity was verified by immunofluorescent staining of the vACE-CREB and vACE-NT infected cells. It takes about three weeks for GFP fluorescence to indicate that about a $100 \%$ of the vACE-GFP cells were infected. At the same time, immunofluorescence analyses (Figure 2) and qPCR ratio of the viral env gene vs. the endogenous RNaseP (not shown) in cells infected with either RCR showed that about $90 \%$ of the cells were infected with either vACE-NT or vACE-CREB.

\section{Knockdown efficiency}

The efficiency of knockdown of CREB in vACECREB infected cells was determined by RT-qPCR and Western blot analyzes relative to cells infected with vACE-NT. Infection with vACE-NT did not change the expression of CREB mRNA and CREB protein significantly relative to the non-infected cells (data not shown) proving that the infection with the retrovirus did not affect the levels of CREB in the infected cells. Therefore, knockdown efficiency by vACE-CREB was compared to cells infected with vACE-NT. Baseline CREB mRNA levels greatly differed between the two cell lines with a 7.6 fold more CREB mRNA in Mel270 cells compared to OMM2.5 cells. Regardless of the initial level of CREB, vACE-CREB knocked down CREB mRNA levels in Mel270 and OMM2.5 to a similar low level (0.18 and 0.21 , respectively) representing a knockdown of $97.4 \%$ and $76.1 \%$, respectively (Figure 3A). The CREB protein levels decreased by $86 \%$ and $56 \%$ in Mel2 70 cells and OMM2.5 cells, respectively (Figure 3B). The minor differences in knockdown efficiencies between the two cell lines may represent differences in the expression of the shRNA and may depend on the initial levels of the target mRNA.

The effect of knockdown on the activity of CREB in the cells was monitored with a luciferase reporter gene plasmid and by measuring the expression of downstream endogenous genes.

Stably infected cells were transfected with a CREmediated luciferase gene expression reporter plasmid, pCREluc. Luciferase activity was determined $48 \mathrm{~h}$ posttransfection. As expected from the initial CREB levels (Figure 3A), the luciferase activity in vACE-NT-infected OMM2.5 cells was $63 \%$ lower than that of Mel270 cells (Figure 4A). Knockdown of CREB resulted in a $36 \%$ reduction of luciferase activity in both cell lines (Figure 4). This result is in correlation with the similar residual levels of CREB in both cell lines ( 0.18 and 0.21 , see above).

Additionally, we measured the expression of the endogenous genes vascular endothelial growth factor (VEGF) and that of proenkephalin (PENK). Knockdown of CREB in Mel270 cells resulted in a $45 \%$ and $32 \%$ reduction of VEGF and PENK mRNAs, respectively, and in a $69 \%$ and $18 \%$, respectively, in OMM2.5 cells (Figure 4B).

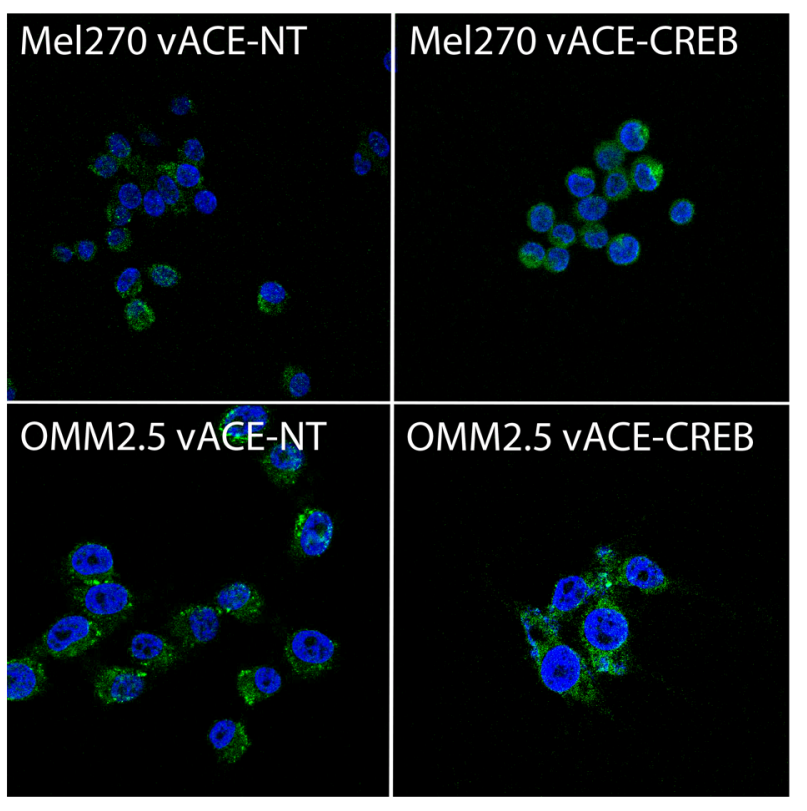

Figure 2: Immunofluorescence assessment of recombinant RCR infectivity. For each slide, Hoechst labeled nuclei (blue) were counted. The staining of viral particles in the cytoplasm of these cells (green) was recorded (x63 magnification). All cells show about a $90 \%$ ratio of green- to blue-labeled cells. 
Effect of hypoxia and of chemotherapeutic drugs on apoptosis and cell viability

The effect of CREB-knockdown on apoptosis in Mel270 and OMM 2.5 cells was determined. At normoxic conditions, CREB knockdown did not increase the apoptotic (sub-G1) fraction (flow cytometry, Table 1, lines 1 and 2) and barely affected cell viability and Caspase 3 activity (cell viability and activation of the Caspase-3 assay, Figure 5).
Before assessing the effect of knockdown of CREB in hypoxia, we verified that the cells respond to the hypoxia, by increasing the expression of Glut- 1 . Indeed, following 48 hours of hypoxia, there is $2.5-2.8$ fold increase in Glut-1 (Figure 6).

In hypoxia, CREB knockdown increased the apoptotic (sub-G1) fraction in both tested cell lines by about 50\% (Table 1, lines 1 and 2).

In the cell viability and Caspase 3 assay, in normoxia, the cell lines grew by $17 \%$ on average after
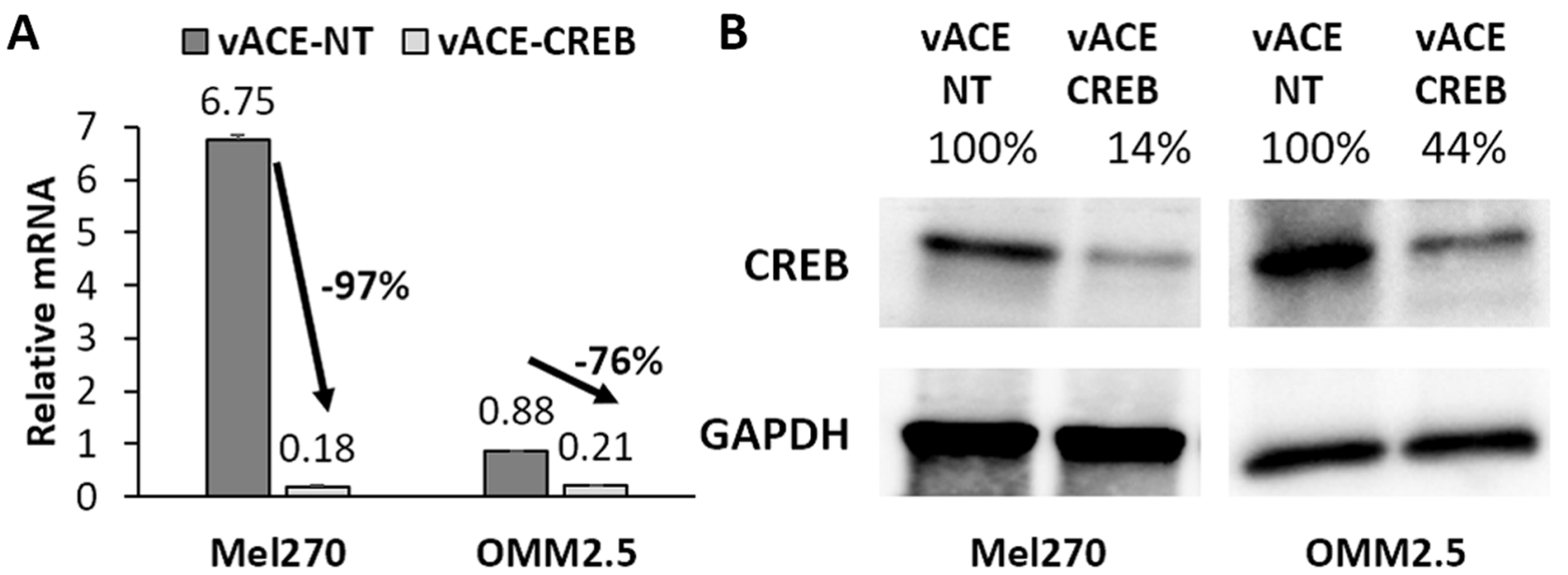

Figure 3: Quantification of the efficiency of knockdown in Mel 270 and OMM2.5 infected cell. The knockdown of CREB in cells fully infected with either vACE-NT or vACE-CREB were analyzed for mRNA and protein levels. (A) Purified mRNA was quantified following RT-qPCR. mRNA levels were normalized to $\beta$-actin mRNA levels in the cells. A knockdown of CREB of $97 \%$ and $76 \%$ was noted in Mel270 and OMM2.5, respectively, $\mathrm{p}<0.05$ (This is a summary of 4 repeats). (B) Western blot analysis of CREB protein ( $43 \mathrm{kDa}$ ). Protein band intensities were compared to those of GAPDH $(37 \mathrm{kDa})$ and to the band intensity of cells infected with vACE-NT (set as $100 \%)$.
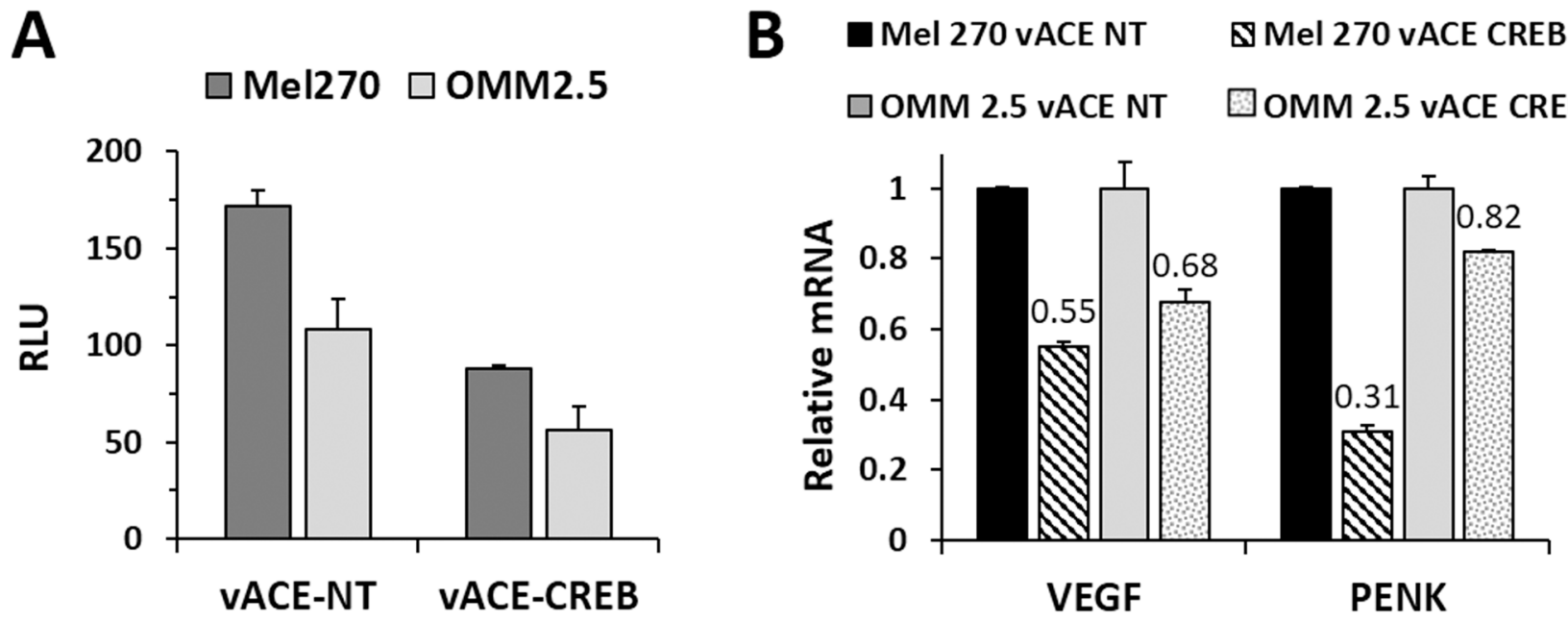

Figure 4: Functional analysis of the effect of CREB knockdown in Mel 270 and OMM2.5 infected cell. The effect on the activity of CREB in the RCR-infected cells was determined in two ways: luciferase reporter gene and knockdown of downstream genes. (A) The infected cells were co-transfected with a CRE-mediated luciferase (luc) reporter plasmid vector, pCREluc together with an expression vector expressing the Renilla luciferase gene, phRLSV40, as a transfection control. The results were normalized to Renilla luciferase activity. (B) Purified mRNA was quantified following RT-qPCR. The levels of mRNA of VEGF and PENK in vACE-CREB infected cells were normalized to $\beta$-actin mRNA levels and to the levels of VEGF and PENK in vACE-NT infected cells, $p<0.05$. 
Table 1: The effect of CREB knockdown, treatment with DOX or DTIC and in combination with apoptosis in normoxia and hypoxia

\begin{tabular}{|c|c|c|c|c|c|c|}
\hline & \multirow[t]{2}{*}{ Cell lines } & \multicolumn{2}{|c|}{ Normoxia } & & \multicolumn{2}{|c|}{ Hypoxia } \\
\hline & & Mel270 & Omm2.5 & & Mel270 & Omm2.5 \\
\hline 1 & No treatment (shNT) & 1 & 1 & Hypoxia/Normoxia & 1 & 1 \\
\hline 2 & shCREB & $\mathrm{NC}$ & $\mathrm{NC}$ & & 1.6 & 1.5 \\
\hline 3 & DOX $0.5 \mu \mathrm{g}$ & $\mathrm{NC}$ & 1.5 & & 1.2 & $\mathrm{NC}$ \\
\hline 4 & DOX $1 \mu \mathrm{g}$ & $\mathrm{NC}$ & 4.0 & & 1.7 & 8.5 \\
\hline 5 & shCREB DOX $0.5 \mu \mathrm{g}$ & 2.2 & 1.2 & & 2.3 & 5.1 \\
\hline 6 & shCREB DOX $1 \mu \mathrm{g}$ & 2.8 & 2.1 & $\begin{array}{c}\text { Hypoxia/ NT in } \\
\text { Hypoxia }\end{array}$ & 2.3 & 22.0 \\
\hline 7 & DTIC $400 \mu \mathrm{g}$ & $\mathrm{NC}$ & $\mathrm{NC}$ & & 2.6 & 1.2 \\
\hline 8 & DTIC $600 \mu \mathrm{g}$ & 1.4 & $\mathrm{NC}$ & & 7.7 & 2.2 \\
\hline 9 & shCREB DTIC $400 \mu \mathrm{g}$ & 1.3 & $\mathrm{NC}$ & & 3.8 & $\mathrm{NC}$ \\
\hline 10 & shCREB DTIC $600 \mu \mathrm{g}$ & 4.6 & 2.5 & & 9.5 & 9.8 \\
\hline
\end{tabular}

The subG1 fraction (apoptotic cells) was defined by FACS analysis. The percent of apoptotic cells in hypoxia (48 $\mathrm{Hr}$ ) in control, cells infected with vACE-NT (Line 1), was calculated relative to apoptotic cells in normoxia. The effect of the different treatments on induction of apoptosis was calculated relative to non-treated cells in normoxia or hypoxia which was set as $100 \%$. NC=No change.

48 hours. Hypoxia of 48 hours inhibited cell growth and barely increased Caspase 3 irrespective of the cell line or CREB level in these cells (note that the activate Caspase 3 measurements were within the background levels compared to the values in Figures 8 and 9). Thus, it seems that unlike HCC cells [14] the two UM cell lines are resistant in vitro to short hypoxia cues (48 hours), and can survive in either normoxia or hypoxia even with highly diminished levels of CREB.

To calibrate the effective concentration of the chemotherapeutic drugs (DOX or DTIC), Mel270 and OMM2.5 were cultured for 24 hours with increasing doses of either drug. A dose-response curve of viable cells post-treatment was plotted to show that both cell lines were sensitive to both chemotherapeutic agents, albeit at different concentrations (Figure 7). These results served us to determine the suboptimal concentration (LD50) of DOX and DTIC that would allow detection of a synergistic effect when we test these chemotherapeutic agents on cells infected with vACE-CREB.

The effect of the selected chemotherapeutic concentrations (DOX 0.5 and $1 \mu \mathrm{g} / \mathrm{ml}$, DTIC 400, $600 \mu \mathrm{g}$ / $\mathrm{ml}$ ) on induction of apoptosis in Mel270 and OMM 2.5 cells is presented in Table 1 (lines 3 and 4 - DOX, lines 7 and $8-$ DTIC). In normoxia, $1 \mu \mathrm{g} / \mathrm{ml}$ of DOX barely increased the apoptotic fraction of Mel270, but in hypoxia, there was a $70 \%$ increase in the apoptotic fraction relative to non-treated cells in hypoxia. These results correlate with the moderate increase in Casp3 in Mel270 cells treated with $1 \mu \mathrm{g} / \mathrm{ml}$ Dox although about $50 \%$ of the cells died at this concentration of DOX (Figure 8). The apoptotic fraction in OMM2.5 increased by about 4-fold and 8 -fold in response to $1 \mu \mathrm{g} / \mathrm{ml}$ of DOX in normoxia and hypoxia, respectively, indicating that OMM2.5 cells are more sensitive than Mel270 to DOX in normoxia and even more so in hypoxia. DTIC barely affected either cell line in normoxia, but there were more than 7- and 2-fold increases in the apoptotic fraction in treated Mel270 and OMM2.5 cells in hypoxia, respectively (Table 1, lines 7-8).

\section{Effectivity of combining treatment with either DOX or DTIC with vACE-CREB}

The combined effect of DOX and CREBknockdown on induction of apoptosis was determined by flow cytometry (Table 1, lines 5-6) where Mel270 cells had a two-fold increase in apoptosis in either normoxia or hypoxia. OMM2.5 also responded with a doubled apoptotic (sub-G1) fraction to treatment with $1 \mu \mathrm{g} / \mathrm{ml}$ concentration of DOX in normoxia, while there was a 22fold increase in the apoptotic fraction to a concentration of $1 \mu \mathrm{g} / \mathrm{ml}$ of DOX in hypoxia.

In the complimentary viability and activation of the Caspase-3 assay, cells not treated with either DOX or DTIC and harboring stably either vACE-NT or vACE-CREB were defined as 100\% (non-treated cells) (Figure 8).

We used the higher dose of DOX for testing the survival and activation of Caspase 3 (Figure 8) and found an about $20 \%$ additive reduction in survival and a 14-26fold increase in activation of Caspase 3 in vACE-CREB 
infected cells vs. vACE-NT infected cells. Thus, the results obtained by the two independent methods correlate with each other.

DTIC is currently used to treat metastatic UM and has not been related directly to CREB activity. We used DTIC to test whether the ability of CREB knockdown to increase the sensitivity of cells to DOX can be generalized to other chemotherapeutic agents. To test if knockdown of CREB increases the cellular sensitivity to DTIC, we used two sub-lethal concentrations of DTIC (400 and $600 \mu \mathrm{g}$ / $\mathrm{ml}$ ). Treatment of vACE-CREB infected Mel270 cells (for 48 hours) with $400 \mu \mathrm{g} / \mathrm{ml}$ hardly affected their apoptotic fraction in normoxia with an almost 4-fold increase in hypoxia. However, use of $600 \mu \mathrm{g} / \mathrm{ml}$ resulted in about 4.5-fold increase in the apoptotic (sub-G1) fraction in normoxic conditions and a 9.5-fold increase in hypoxic conditions (Table 1, lines 9-10). OMM2.5 cells were less sensitive than Mel270 cells to treatment with DTIC even in reduced CREB conditions. vACE-CREB infected OMM2.5 cells did not respond to $400 \mu \mathrm{g} / \mathrm{ml}$ of DTIC in either normoxia or hypoxia. However, there was a twofold increase in apoptosis with $600 \mu \mathrm{g} / \mathrm{ml}$ of DTIC in normoxia and a 9.8-fold increase in apoptosis in hypoxia.

The complementary analysis of survival and Caspase 3 activation showed an additive $20-40 \%$ reduction in survival with $400 \mu \mathrm{g} / \mathrm{ml}$ and a $16-32 \%$ additive reduction with $600 \mu \mathrm{g} / \mathrm{ml}$ (where the drug itself is more toxic) with a parallel additive increase in the activation of

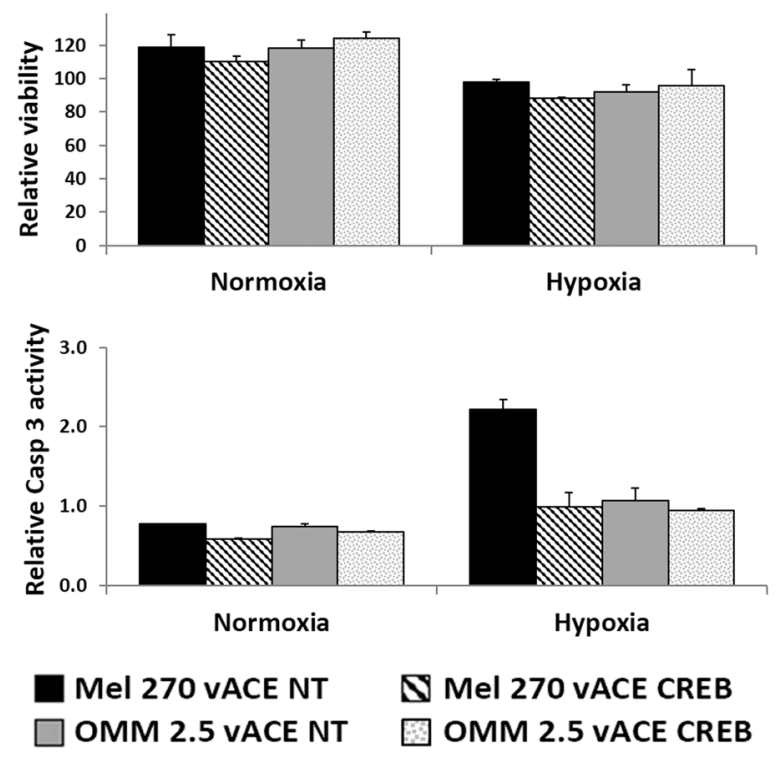

Figure 5: The effect of CREB on uveal melanoma's sensitivity to hypoxia. Mel270 and OMM2.5 cell lines infected with either vACE-NT or vACE-CREB were cultivated in normoxia and hypoxia for 48 hours before the viability (top) and the activation of Caspase 3 (bottom) were determined by the Fluorescent Cell Viability and Caspase-Glo 3/7 Assay (Promega) (expressed in relative light units). There was no significant difference between the viability measurements, and the activation of Caspase 3 measurements were within the background levels.

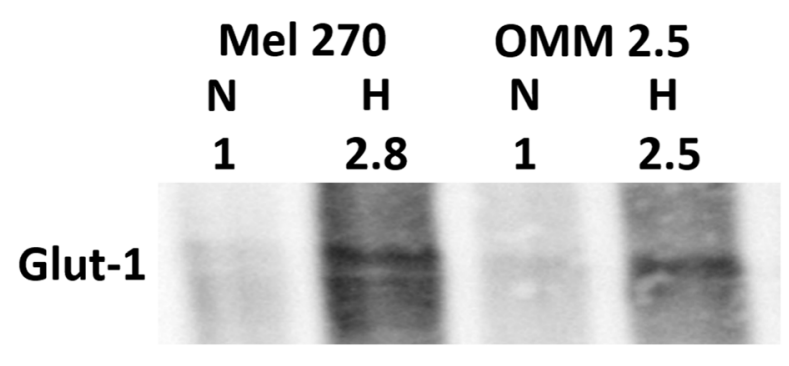

GAPDH

Figure 6: Cellular response to hypoxia. Mel270 and OMM2.5 cell lines were cultivated in normoxic (N) and hypoxic (H) conditions for 48 hours and the relative expression of Glut-1 $(50 \mathrm{kDa})$ was determined by Western blot analysis. Band intensities were normalized to GAPDH (37 kDa), and the intensities of hypoxia were normalized to those in normoxia. 
Caspase 3 (Figure 9). Comparison of the killing effect of DTIC on vACE-NT infected cells in hypoxia (Figure 9) vs. normoxia (Figure 8) shows that DTIC is less effective in hypoxia. Taking into account the above findings that these UM cells are insensitive to hypoxia, the additive killing noted in the vACE-CREB infected cells in hypoxia suggests that CREB activity protects cells from DTIC in hypoxia.

\section{DISCUSSION}

Solid tumors grow faster than they can attract blood vessels into them generating an ongoing formation of hypoxic regions within tumors. In these regions, tumor cells are too far from the present vessels, and thus oxygen and drugs do not reach them. In previous work, we have shown that knockdown of CREB prevents the cellular responses to hypoxia in hepatocellular carcinoma, increase cell death in hypoxia, and lead to increased sensitivity to treatment with doxorubicin in normoxia and hypoxia, in vitro and in vivo [14].

Based on these results we investigated whether infective knockdown of CREB by the retroviral replication competent vector will be effective on other tumors.

Metastatic uveal melanoma remains a therapeutic challenge with no effective chemotherapeutic or biologic treatment. In this work, we present a novel combinatorial system that may open an avenue for effective treatment for mUM.

As illustrated in Table 1 (line 1) and Figure 5, uveal melanoma cell lines are insensitive to hypoxia. Since we demonstrated that CREB plays a pivotal role in the cellular responses to hypoxia, we knocked down CREB in two UM cell lines and found a 50\% increase in the apoptotic fraction following knockdown of CREB (Table 1, line 2). Thus, CREB is involved in the resistance to hypoxia cue in these cell lines, although the increase in the apoptotic fraction was not expressed in cell death (Figure 5). This discrepancy may result from the different sensitivities of flow cytometry (apoptotic fraction) vs. the Fluorescent Cell Viability and Caspase-Glo 3/7 Assay. These results may suggest that Caspase 3 is not involved in the generation of the sub-G1 fraction. Additionally, the gap between the increase in apoptosis and the lack of increase in cell death reflects the higher resistance of UM cells to hypoxia relative to HCC cells.

Knockdown of CREB resulted in a decrease in the expression of VEGF in the UM cells (Figure 4), similar to our findings in HCC [14], which could contribute to a decrease in tumor cell growth in vivo due to inhibition of perfusion of the growing tumors and increase the hypoxic areas within the tumors. Thus, infection with vACE-CREB will, on the one hand, increase the hypoxic regions, and on the other hand, prevent the tumor cells from responding to the hypoxia cue.

Cell viability in response to chemotherapy of 77 choroidal melanomas and 58 cutaneous melanomas was assessed using an ex vivo ATP-based chemosensitivity assay (ATP-TCA) [4]. Of 12 different chemotherapeutic agents and one of two combinations, almost none of the UMs responded to the chemotherapy. DTIC is in use in some centers to treat mUM with a limited success rate [25, 26]. DOX, has been used to treat primary liver tumors, which has not been used for mUM in which almost a $100 \%$ of the patients present with liver metastases. In this work we found that two separate UM cell lines responded to these two agents, DTIC and DOX (Figures 8 and 9), albeit not equally so.

There could be several explanations for the effect we found versus Naele's results. One would be that we used different concentrations of the drugs. Another explanation is that these cell lines represent the outliers that do respond
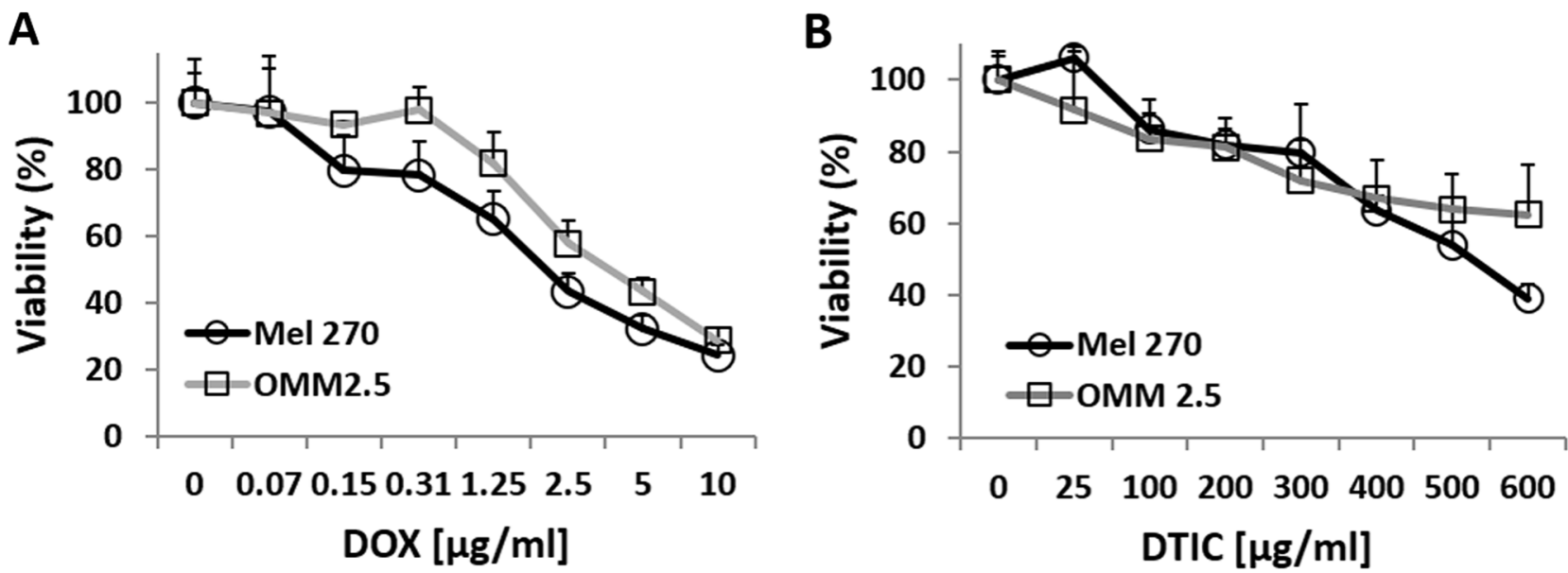

Figure 7: Dose response to treatment of UM cells with DOX or DTIC. Mel270 and OMM2.5 cell lines were cultivated in normoxic conditions and treated with various concentrations of either DOX (A) or DTIC (B) for 48 hours and viability of the cells was determined by the Fluorescent Cell Viability Assay (Promega). 
to chemotherapy. The world of oncology is moving in the direction of personalized medicine as we learn more about inter-patient variations, inter-tumor variability, and how different patients with what was thought of as the same tumor respond differently to chemotherapy [27]. The implication is that outliers should not be ignored and that these two cell lines may represent at least a small group of UM patients. However, the findings of this study indicate that a medication, DOX, that was previously disregarded may enter our armamentarium against metastatic UM.

DOX is known to activate Caspase 3 [28]. Knockdown of CREB increased the DOX-induced activation of Caspase 3 (Figures 8 and 9) resulting in an about $70 \%$ increase in cell death in both $\mathrm{O}_{2}$ conditions, and in an increase in the apoptotic fraction (Table 1). A similar beneficial effect was measured when we combined treatment with DTIC and CREB knockdown.

DOX has a limited therapeutic window due to its toxic effect on normal tissues, with the most adversely affected organ being the heart [29]. We hypothesize that the increase in cell sensitivity to DOX, resulting from CREB knockdown, may result in a decrease in the required active dose of the drug and thus to a reduction in DOX cardiotoxicity.

Latorre et al. used DNA and aptamer stabilized gold nanoparticles (GNP) for targeted delivery of anticancer therapeutics. They used GNPs to deliver DOX to cancer cells, including two uveal melanoma cell lines: OMM1.3 and Mel202 [22]. Dong et al. used a conjugate of polyethyleneimine (PEI) with DOX via a $\mathrm{pH}$-responsive hydrazone linkage (PEI-Hz-DOX, PHD) and a tumor-targeting folate ligand conjugated to PEI using polyethylene glycol (PEG) as a linker (PEI-PEGFolate, PPF) in tandem with siRNA targeting vascular endothelial growth factor (VEGF) [30]. They used these nanocomplexes to deliver DOX and the siRNA to breast cancer cells. They found a synergistic effect of the use of DOX and siRNA for VEGF [30]. These results are in agreement with our findings described above. Knockdown of CREB increased the sensitivity to DOX (Table 1 and Figures 8 and 9) and could allow the use of lower concentrations of DOX to avoid its toxicity in
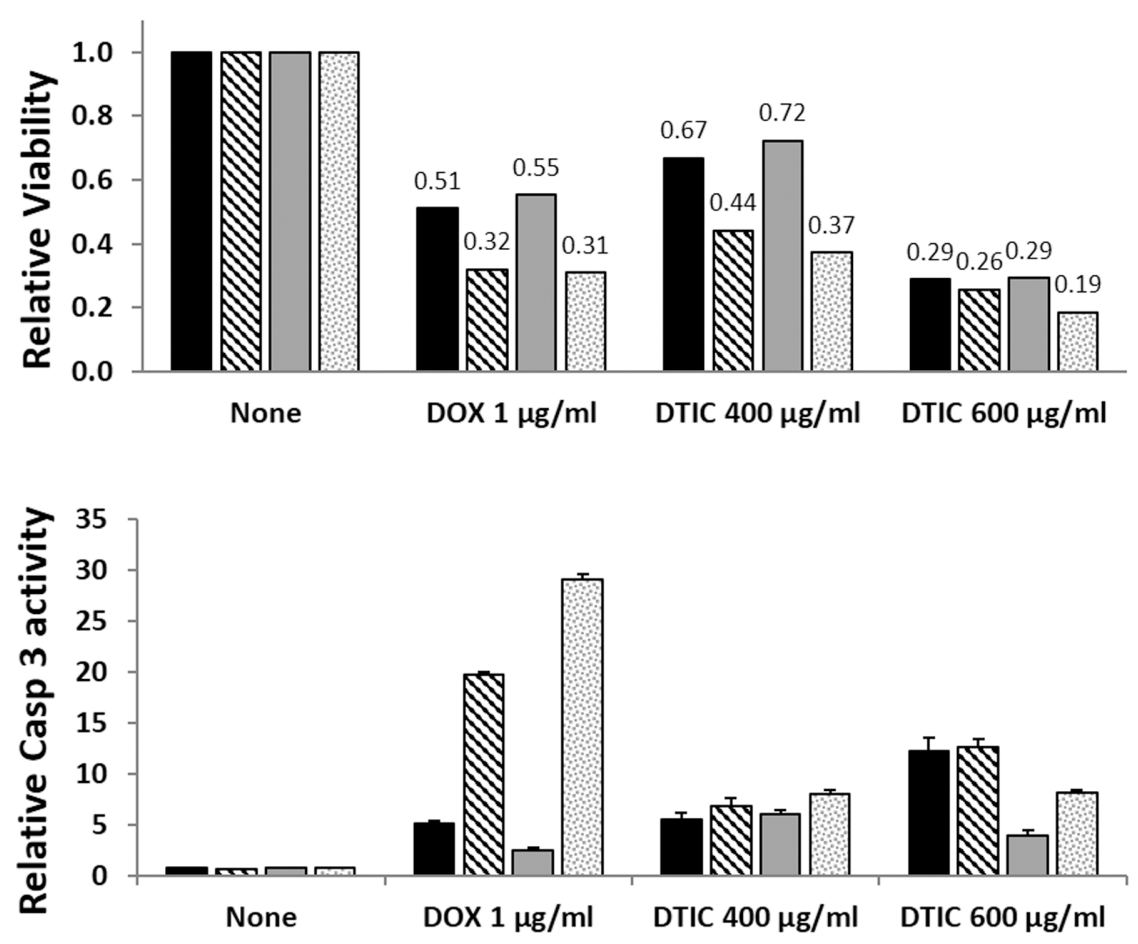

\section{Mel 270 vACE NT $\$$ Mel 270 vACE CREB OMM 2.5 vACE NT 圆 OMM 2.5 vACE CREB}

Figure 8: The effect of CREB on uveal melanoma's sensitivity to chemotherapy in normoxia. Mel270 and OMM2.5 cell lines infected with either vACE-NT or vACE-CREB were cultivated in normoxic conditions and treated with either DOX $(1 \mu \mathrm{g} / \mathrm{ml}) \mathrm{or}$ DTIC $(400 \mu \mathrm{g} / \mathrm{ml}$ or $600 \mu \mathrm{g} / \mathrm{ml}$ ) for 48 hours and viability (top) and activation of Caspase 3 (bottom), were determined relative to non-treated vACE-NT and vACE-CREB infected cells (set as $100 \%$ for each infected cell). 
patients. Moreover, the unique properties of our system in which the MuLV-based RCR recombinant vectors which generate an ongoing infectious knockdown of CERB in tumor growing cells have specificity to tumor cells more than the above-mentioned approaches of Latorre and Dong.

In this work we demonstrated that the RCR vector expressing shRNA could infect UM cell lines and spread efficiently to knock down the expression of CREB in these cells, resulting in diminished expression of downstream CREB-mediated genes (Figure 4). This knockdown did not affect cell viability under normal growth conditions. However, when infected cells were treated with sub-optimal doses of DOX, the sensitivity to the drug doubled for both cell lines in normoxia. In hypoxia, the sensitivity of Mel270 doubled, and that of OMM2.5 increased dramatically (by 22-fold). Despite the less efficient knockdown of CREB in OMM2.5 vs. Mel270 (Figure 3), OMM2.5 showed a higher dependence on CREB in their response to DOX.
Unlike DOX, DTIC is used in the treatment of metastatic UM, but the efficacy of this drug is far from optimal [31]. In this work, we found that knocking down CREB increases the sensitivity of Mel270 and less so of OMM2.5 to DTIC. This demonstrates the variability in response to chemotherapy of tumor cells from different origins again. The results presented here bring hope that infection with vACE-CREB can increase the sensitivity of some of the tumors to DTIC or DOX and by which, increase the treatment efficacy.

In summary, we have shown that UM can respond to a well-known chemotherapeutic agent (DOX) that was thought to be ineffective for this disease. We have shown that knocking down CREB can increase the sensitivity of some UMs to DOX. The increased sensitivity also to DTIC may indicate that combining CREB knockdown with chemotherapeutic agents may be a general mechanism to improve the sensitivity of solid tumors to chemotherapy.
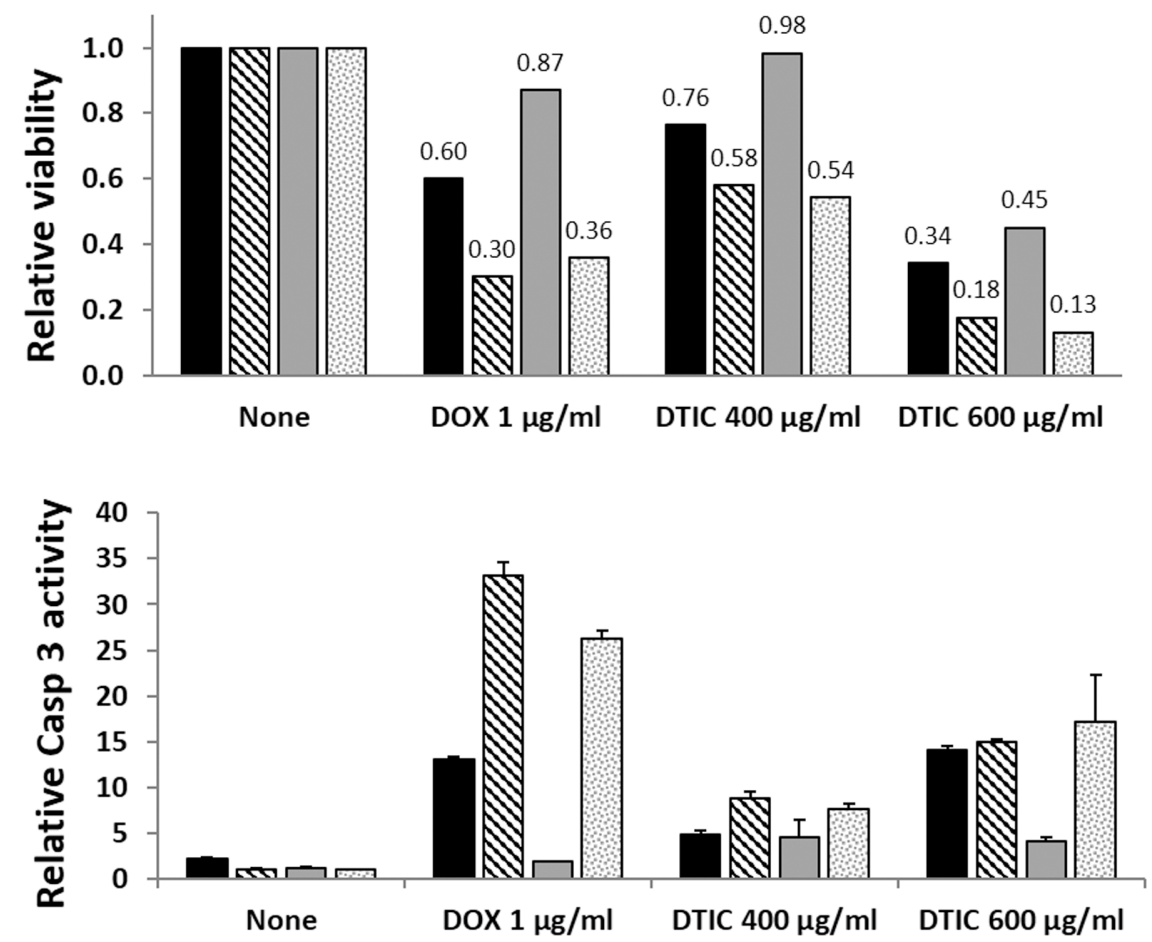

Mel 270 vACE NT $\square$ OMM 2.5 VACE NT $\triangle$ Mel 270 vACE CREB OMM 2.5 VACE CREB

Figure 9: The effect of CREB on uveal melanoma's sensitivity to chemotherapy in hypoxia. Mel270 and OMM2.5 cell lines infected with either vACE-NT or vACE-CREB were cultivated in hypoxic conditions and treated with either DOX $(1 \mu \mathrm{g} / \mathrm{ml})$ or DTIC $(400 \mu \mathrm{g} / \mathrm{ml}$ or $600 \mu \mathrm{g} / \mathrm{ml})$ for 48 hours. Viability (top) and activation of Caspase 3 (bottom), were normalized to non-treated vACE-NT and vACE-CREB infected cells (set as 100\% for each infected cell) and expressed in relative light units. 


\section{MATERIALS AND METHODS}

\section{Cell culture}

Human UM OMM2.5 [32] and Mel270 cell lines [33] (verified by STR analysis and a kind gift from Prof. Sarah Coupland, Liverpool, UK) were grown in RPMI supplemented with $10 \%$ fetal bovine serum, $2 \mathrm{mM}$ glutamine, $100 \mathrm{IU} / \mathrm{ml}$ penicillin, $100 \mu \mathrm{g} / \mathrm{ml}$ streptomycin (Biological Industries) and incubated at $37^{\circ} \mathrm{C}$ in a humidified atmosphere with $5 \% \mathrm{CO}_{2}$.

\section{Plasmids and viruses}

The plasmid pACE-GFP (a kind gift from Prof. Noriyuki Kasahara, Los Angeles, California [23]) contains a full-length replication-competent amphotropic $\mathrm{MuLV}$ provirus with an additional internal ribosome entry site (IRES)-GFP cassette flanked by BsiWI and NotI restriction enzymes sites. This cassette was replaced by oligonucleotides harboring the $\mathrm{H} 1$ promoter driving the transcription of the following shRNA sequences:

5' GAGAGAGGTCCGTCTAATGTTCAAGAGA CATTAGACGGACCTCTCTCTTTTT (pACE-CREB). $5^{\prime}$ ACCAAGATGAAGAGCACCAACCTGAACCATT GGTGCTCTTCATCTTGGTTTTTTT (pACE-NT. Nontarget shRNA). See Figure 1 for a schematic presentation of the vectors.

\section{Virus preparation}

HEK293T cells were transiently transfected with either one of the pACE plasmids, described above and in Figure 1, using FuGENE HD reagent (Promega). The medium containing the virus particles was harvested $48 \mathrm{~h}$ later, filtered (MILLEX-HV, PVDF $0.45 \mu$ ) and stored at $-80^{\circ} \mathrm{C}$.

\section{Viral titer and spread}

Virus titer was determined by qPCR (see Results section) comparing RNase $\mathrm{P}$ to the viral env gene. Virus spread was determined either by flow cytometry of cells infected with vACE-GFP or by qPCR comparing RNase P to viral env gene at each time point.

\section{Immunofluorescence}

RCR-infected cells were grown on chamber slides for three days. Slides were fixed with $4 \%$ PFA for 2 minutes followed by incubation with pre-cooled $100 \%$ methanol for 5 minutes. The slides were washed three times with PBS, blocked with 3\% BSA for 30 minutes and incubated for 45 minutes with a primary antibody targeting the p-30 of MuLV driven from R187 cells (ATCC CRL-1912). The slides were washed three times with PBS, incubated with a fluorescent secondary antibody (alexaflour-488 AB-150153 donkey-anti-rat, Abcam) for 45 minutes, and washed three more times with PBS. The nuclei were stained with Hoechst. Stained slides were mounted with Vectashield (Vector Laboratories). Slides were scanned with a Zeiss LSM 710 microscope at x63 magnification and analyzed with ZEN2010 software (Zeiss). Cells were counted for each slide by the number of Hoechst stained nuclei. The ratio of infected cells was calculated for each slide.

\section{Quantitative real-time PCR}

RNA was extracted from the cells using the SV Total RNA Isolation System (Promega), according to the manufacturer's instructions. The purified RNA samples were subjected to reverse transcription using GoScript (Promega), monitored by quantitative $7900 \mathrm{HT}$ real-time PCR apparatus (Applied Biosystems) utilizing the GoTaq Real-Time PCR reagents (Promega) and the specific primers: CREB: fp- 5'_CCCAGCACTTCCTACACAGCCTGC, rp5' CGAGCTGCTTCCUGTTCTTCATTAGACG. The results were normalized to the cellular house-keeping gene GAPDH: fp-5' CCATCTTCCAGGAGCGAGATCC, rp5'_GCAAATGAGCCCCAGCTTCTCC.

\section{Western blot analysis}

Western Blot analyzes were carried out by standard procedure [34]. Briefly, equal amounts of total protein were prepared in Laemmli SDS loading buffer, resolved by $10 \%$ SDS-PAGE electrophoresis and transferred to PVDF membranes (Millipore). Specific proteins were detected with either CREB, Glut-1 or GAPDH primary antibodies (Abcam) and secondary HRP-conjugated antibody (Promega). The proteins were visualized using the enhanced chemiluminescence (ECL) system (Promega), scanned by the MiniBIS Pro (DNR) scanner and band intensities were quantified by utilizing TINA 20 program (Raytest) on pictures without overexposure of any of the bands.

\section{Luciferase assay}

Cells fully infected with vACE-CREB or vACE NT were seeded in 6-well plates at a concentration of 500,000 cells/well for 24 hours. The infected cells were cotransfected ( $3 \mu \mathrm{g}$ DNA) with the CRE-mediated luciferase (luc) reporter plasmid vector, pCREluc, together with $0.25 \mu \mathrm{g}$ of an expression vector expressing the Renilla luciferase gene, phRLSV40, as a transfection control (Promega Corp) using FuGENE HD (Promega Corp). Luciferase activity was determined $48 \mathrm{~h}$ post-transfection, according to the manufacturer's instructions (DualLuciferase reporter assay system, Promega Corp) by an automatic Mithras LB 940 photoluminometer (Berthold Technologies, Oak Ridge, TN, USA). The results were normalized to Renilla luciferase activity. 


\section{Flow cytometry}

Stably infected cells with either one of the recombinant RCRs mentioned above were cultivated in normoxic and hypoxic conditions $\left(1 \% \mathrm{O}_{2}\right)$ with and without a variety of concentrations of either DOX or DTIC for $48 \mathrm{~h}$. Cells were subjected to cell cycle analysis by Eclipse- Analyzer.

\section{Cell viability and activation of Caspase-3}

Stably infected cells with either vACE-NT or vACE CREB were cultivated at a concentration of 10,000 cells/ well in 96-well plates (4 repeats) at normoxia or hypoxia $\left(0.5 \% \mathrm{O}_{2}\right.$, in hypoxia boxes) for up to 48 hours. Cell viability and Caspase-3 activity were determined by the Fluorescent Cell Viability and Caspase-Glo 3/7 Assay (Promega) according to the manufacturer's instructions. For the experiments with the chemotherapeutic agents, cells were cultivated with varying concentrations of either DOX or DTIC for 48 hours before determining viability and Caspase-3 activity.

\section{Statistical analysis}

Statistical analysis was performed with JMP 9.0 (SAS). Analysis of variance (ANOVA) was used to compare mRNA and protein levels, survival rates and the Caspase 3 activation levels.

\section{Abbreviations}

UM: uveal melanoma; mUM: metastatic uveal melanoma; DTIC: dacarbazine; DOX: doxorubicin; RCR: replicative competent retroviruses; AMP: adenosine monophosphate; CRE: cyclic AMP-response-element; CREB: CRE binding protein; shRNA: short hairpin RNA; MuLV: Murine leukemia virus; NT: non-target; HCC: hepatocellular carcinoma; GNPs: gold nanoparticles; HIF: hypoxia inducible factor; IRES: internal ribosome entry site; qPCR: quantitative real-time PCR; VEGF: vascular endothelial growth factor; PENK: proenkephalin.

\section{Author contributions}

All the authors have directly participated in the preparation of this manuscript and have approved the final version submitted. DS performed most of the experiments. ST, HV, and MG helped with the experiments. JP and NC gave critical review notes. $\mathrm{AH}$ and $\mathrm{SF}$ were responsible for designing the study, funding it and writing the manuscript.

\section{CONFLICTS OF INTEREST}

The other authors declare no competing interests.

\section{FUNDING}

This work was supported by grants from the Israeli Science Foundation (1935/12), a grant from the Ministry of Science, Technology and Space, Israel, and the Ministère de l'Education Nationale, de l'Enseignement Supérieur et de la Recherche, France, and the Israel Cancer Association (20150029). The funders had no role in the study design, data collection and analysis, decision to publish or preparation of the manuscript.

\section{REFERENCES}

1. Kujala E, Makitie T, Kivela T. Very long-term prognosis of patients with malignant uveal melanoma. Invest Ophthalmol Vis Sci. 2003; 44:4651-9.

2. Diener-West M, Reynolds SM, Agugliaro DJ, Caldwell R, Cumming K, Earle JD, Hawkins BS, Hayman JA, Jaiyesimi I, Jampol LM, Kirkwood JM, Koh WJ, Robertson DM, et al. Development of metastatic disease after enrollment in the COMS trials for treatment of choroidal melanoma: collaborative ocular melanoma study group report no. 26. Arch Ophthalmol. 2005; 123:1639-43. https://doi. org/10.1001/archopht.123.12.1639.

3. Augsburger JJ, Correa ZM, Shaikh AH. Effectiveness of treatments for metastatic uveal melanoma. Am J Ophthalmol. 2009; 148:119-27. https://doi.org/10.1016/j. ajo.2009.01.023.

4. Neale MH, Myatt NE, Khoury GG, Weaver P, Lamont A, Hungerford JL, Kurbacher CM, Hall P, Corrie PG, Cree IA. Comparison of the ex vivo chemosensitivity of uveal and cutaneous melanoma. Melanoma Res. 2001; 11:601-9.

5. Hanahan D, Weinberg RA. The hallmarks of cancer. Cell. $2000 ; 100: 57-70$.

6. Danial NN, Korsmeyer SJ. Cell death: critical control points. Cell. 2004; 116:205-19.

7. Mooy CM, Luyten GP, de Jong PT, Luider TM, Stijnen T, van de Ham F, van Vroonhoven CC, Bosman FT. Immunohistochemical and prognostic analysis of apoptosis and proliferation in uveal melanoma. Am J Pathol. 1995; 147:1097-104.

8. Jay V, Yi Q, Hunter WS, Zielenska M. Expression of bcl-2 in uveal malignant melanoma. Arch Pathol Lab Med. 1996; $120: 497-8$

9. Coupland SE, Bechrakis N, Schuler A, Anagnostopoulos I, Hummel M, Bornfeld N, Stein H. Expression patterns of cyclin D1 and related proteins regulating G1-S phase transition in uveal melanoma and retinoblastoma. Br J Ophthalmol. 1998; 82:961-70.

10. Chana JS, Wilson GD, Cree IA, Alexander RA, Myatt N, Neale M, Foss AJ, Hungerford JL. c-myc, p53, and Bcl-2 expression and clinical outcome in uveal melanoma. Br J Ophthalmol. 1999; 83:110-4. 
11. Lawry J, Currie Z, Smith MO, Rennie IG. The correlation between cell surface markers and clinical features in choroidal malignant melanomas. Eye (Lond). 1999; 13:301-8. https://doi.org/10.1038/eye.1999.79.

12. Nemati F, Sastre-Garau X, Laurent C, Couturier J, Mariani P, Desjardins L, Piperno-Neumann S, Lantz O, Asselain B, Plancher C, Robert D, Peguillet I, Donnadieu MH, et al. Establishment and characterization of a panel of human uveal melanoma xenografts derived from primary and/or metastatic tumors. Clin Cancer Res. 2010; 16:2352-62. https://doi.org/10.1158/1078-0432.CCR-09-3066.

13. Abramovitch R, Tavor E, Jacob-Hirsch J, Zeira E, Amariglio N, Pappo O, Rechavi G, Galun E, Honigman A. A pivotal role of cyclic AMP-responsive element binding protein in tumor progression. Cancer Res. 2004; 64:1338-46.

14. Shneor D, Folberg R, Pe'er J, Honigman A, Frenkel S. Stable knockdown of CREB, HIF-1 and HIF-2 by replication-competent retroviruses abrogates the responses to hypoxia in hepatocellular carcinoma. Cancer Gene Ther. 2017; 24:64-74. https://doi.org/10.1038/ cgt.2016.68.

15. Park SI, Park SJ, Lee J, Kim HE, Sohn JW, Park YG. Inhibition of cyclic AMP response element-directed transcription by decoy oligonucleotides enhances tumor-specific radiosensitivity. Biochem Biophys Res Commun. 2016; 469:363-9. https://doi.org/10.1016/j. bbrc.2015.11.122.

16. Balogh A, Nemeth M, Koloszar I, Marko L, Przybyl L, Jinno K, Szigeti C, Heffer M, Gebhardt M, Szeberenyi J, Muller DN, Setalo G Jr, Pap M. Overexpression of CREB protein protects from tunicamycin-induced apoptosis in various rat cell types. Apoptosis. 2014; 19:1080-98. https:// doi.org/10.1007/s10495-014-0986-z.

17. Wang P, Huang S, Wang F, Ren Y, Hehir M, Wang X, Cai J. Cyclic AMP-response element regulated cell cycle arrests in cancer cells. PLoS One. 2013; 8:e65661. https://doi. org/10.1371/journal.pone.0065661.

18. Lee BS, Kim SH, Jin T, Choi EY, Oh J, Park S, Lee SH, Chung JH, Kang SM. Protective effect of survivin in Doxorubicin-induced cell death in h9c2 cardiac myocytes. Korean Circ J. 2013; 43:400-7. https://doi.org/10.4070/ kcj.2013.43.6.400.

19. Sayan M, Shukla A, MacPherson MB, Macura SL, Hillegass JM, Perkins TN, Thompson JK, Beuschel SL, Miller JM, Mossman BT. Extracellular signal-regulated kinase 5 and cyclic AMP response element binding protein are novel pathways inhibited by vandetanib (ZD6474) and doxorubicin in mesotheliomas. Am J Respir Cell Mol Biol. 2014; 51:595-603. https://doi.org/10.1165/ rcmb.2013-0373TR.

20. Tacar O, Sriamornsak P, Dass CR. Doxorubicin: an update on anticancer molecular action, toxicity and novel drug delivery systems. J Pharm Pharmacol. 2013; 65:157-70. https://doi.org/10.1111/j.2042-7158.2012.01567.x.
21. Brasiuniene B, Sokolovas V, Brasiunas V, Barakauskiene A, Strupas K. Combined treatment of uveal melanoma liver metastases. Eur J Med Res. 2011; 16:71-5.

22. Latorre A, Posch C, Garcimartin Y, Celli A, Sanlorenzo M, Vujic I, Ma J, Zekhtser M, Rappersberger K, Ortiz-Urda S, Somoza A. DNA and aptamer stabilized gold nanoparticles for targeted delivery of anticancer therapeutics. Nanoscale. 2014; 6:7436-42. https://doi.org/10.1039/c4nr00019f.

23. Logg CR, Tai CK, Logg A, Anderson WF, Kasahara N. A uniquely stable replication-competent retrovirus vector achieves efficient gene delivery in vitro and in solid tumors. Hum Gene Ther. 2001; 12:921-32. https://doi. org/10.1089/104303401750195881.

24. Kizaka-Kondoh S, Inoue M, Harada H, Hiraoka M. Tumor hypoxia: a target for selective cancer therapy. Cancer Sci. 2003; 94:1021-8.

25. Carvajal RD, Schwartz GK, Mann H, Smith I, Nathan PD. Study design and rationale for a randomised, placebocontrolled, double-blind study to assess the efficacy of selumetinib (AZD6244; ARRY-142886) in combination with dacarbazine in patients with metastatic uveal melanoma (SUMIT). BMC Cancer. 2015; 15: 467. https:// doi.org/10.1186/s12885-015-1470-z.

26. Schinzari G, Rossi E, Cassano A, Dadduzio V, Quirino M, Pagliara M, Blasi MA, Barone C. Cisplatin, dacarbazine and vinblastine as first line chemotherapy for liver metastatic uveal melanoma in the era of immunotherapy: a single institution phase II study. Melanoma Res. 2017; 27:591-5. https://doi.org/10.1097/CMR.0000000000000401.

27. Lazar V, Rubin E, Depil S, Pawitan Y, Martini JF, GomezNavarro J, Yver A, Kan Z, Dry JR, Kehren J, Validire P, Rodon J, Vielh P, et al. A simplified interventional mapping system (SIMS) for the selection of combinations of targeted treatments in non-small cell lung cancer. Oncotarget. 2015; 6:14139-52. https://doi.org/10.18632/oncotarget.3741.

28. Shaker RA, Abboud SH, Assad HC, Hadi N. Enoxaparin attenuates doxorubicin induced cardiotoxicity in rats via interfering with oxidative stress, inflammation and apoptosis. BMC Pharmacol Toxicol. 2018; 19: 3. https:// doi.org/10.1186/s40360-017-0184-z.

29. Tacar O, Dass CR. Doxorubicin-induced death in tumour cells and cardiomyocytes: is autophagy the key to improving future clinical outcomes? J Pharm Pharmacol. 2013; 65:1577-89. https://doi.org/10.1111/jphp.12144.

30. Dong D, Gao W, Liu Y, Qi XR. Therapeutic potential of targeted multifunctional nanocomplex co-delivery of siRNA and low-dose doxorubicin in breast cancer. Cancer Lett. 2015; 359:178-86. https://doi.org/10.1016/j. canlet.2015.01.011

31. Carvajal RD, Sosman JA, Quevedo JF, Milhem MM, Joshua AM, Kudchadkar RR, Linette GP, Gajewski TF, Lutzky J, Lawson DH, Lao CD, Flynn PJ, Albertini MR, et al. Effect of selumetinib vs chemotherapy on progressionfree survival in uveal melanoma: a randomized clinical 
trial. JAMA. 2014; 311:2397-405. https://doi.org/10.1001/ jama.2014.6096.

32. Luyten GP, Naus NC, Mooy CM, Hagemeijer A, KanMitchell J, Van Drunen E, Vuzevski V, De Jong PT, Luider TM. Establishment and characterization of primary and metastatic uveal melanoma cell lines. Int J Cancer. 1996; 66:380-7. https://doi.org/10.1002/ (SICI)1097-0215(19960503)66:3<380::AID-

IJC19>3.0.CO;2-F.
33. Verbik DJ, Murray TG, Tran JM, Ksander BR. Melanomas that develop within the eye inhibit lymphocyte proliferation. Int J Cancer. 1997; 73:470-8.

34. Meyuhas R, Pikarsky E, Tavor E, Klar A, Abramovitch R, Hochman J, Lago TG, Honigman A. A Key role for cyclic AMP-responsive element binding protein in hypoxia-mediated activation of the angiogenesis factor CCN1 (CYR61) in Tumor cells. Mol Cancer Res. 2008; 6:1397-409. https://doi. org/10.1158/1541-7786.MCR-07-2086. 